\title{
Low $Q^{2}$ wave-functions of pions and kaons and their parton distribution functions.
}

\author{
C. Avila*, J. Magnin ${ }^{\dagger}$ and J.C. Sanabria ${ }^{\ddagger}$ \\ Depto. de Física, Universidad de los Andes, \\ AA 4976, Santafé de Bogotá, Colombia
}

November 14, 2018

\begin{abstract}
We study the low $Q^{2}$ wave-functions of pions and kaons as an expansion in terms of hadron-like Fock state fluctuations. In this formalism, pion and kaon wave-functions are related one another. Consequently, the knowledge of the pion structure allows the determination of parton distributions in kaons. In addition, we show that the intrinsic (low $Q^{2}$ ) sea of pions and kaons are different due to their different valence quark structure. Finally, we analize the feasibility of a method to extract kaon's parton distribution functions within this approach and compare with available experimental data.
\end{abstract}

\section{Motivation}

At present the only information available on the structure of unstable mesons is on parton distributions functions (pdf) in pions. These pdf are extracted from Drell-Yan (D-Y) dilepton and prompt photon production in pion-nucleon interactions.

In principle, the same type of experiments can be used to extract information on parton distributions in kaons by only replacing the pion beam by a kaon one. However, this requires high intensity kaon beams which are not easily attainable in present day experiments. Furthermore, there is an additional difficulty which is inherent to the kaon structure because strange valence quarks in the kaon must anihilate with strange quarks in the target particles. As targets are made of nucleons, and strange quarks are in the sea of them, these $s-\bar{s}$ anihilation processes are expected to have a small

\footnotetext{
*e-mail: cavila@uniandes.edu.co

†e-mail: jmagnin@uniandes.edu.co

‡e-mail: jsanabri@uniandes.edu.co
} 
contribution to the total D-Y dilepton cross section. Consequently, it is very difficult to have a precise measurement of the strange valence quark distribution in kaons. Other procedures involving the detection of a D-Y dilepton pair accompanied by a fast pion in the final state have been proposed to measure the strange quark distribution in kaons [1], but they rely on several assumptions coming from recombination models.

The situation, however, is radically different with the light valence quark distribution. In fact, the $\bar{u}_{K}$ distribution can be measured rather well in D-Y experiments since this is, by far, the major contribution to $q \bar{q} \rightarrow l^{+} l^{-}$processes in $K^{-}-$nucleon interactions. The first, and up to our knowledge, the only attempt in this direction was made by the NA3 Collaboration [2], who measured the ratio of the $u$-quark distribution in kaons to the $u$-quark distribution in pions, $\bar{u}_{K} / \bar{u}_{\pi}$. In this experiment, the ratio $\bar{u}_{K} / \bar{u}_{\pi}$ was extracted from D-Y dimuon production in $150 \mathrm{GeV} / c$ $K^{-}, \pi^{-}-$nucleus interactions. The measurement is, however, subject to large uncertainties due to the limited statistic of the experiment and several assumptions made in the calculation of the $\bar{u}_{K} / \bar{u}_{\pi}$ ratio.

Another possibility is to extract information on the kaon structure from precise measurements of the pion's pdf. To achieve this end, a model of the low $Q^{2}$ wavefunction of hadrons in terms of hadron-like Fock state fluctuations is called for. In this model, as we will show, the pion and kaon low $Q^{2}$ wave-functions are related to each other. This allows in principle to extract information on the low $Q^{2}$ structure of kaons from the pion structure by means of a rather direct procedure. It is worth to note that this procedure only needs the measurement of the pion's pdf and the ratio $\bar{u}_{K} / \bar{u}_{\pi}$, avoiding in this way any measurement of the strange distribution in kaons, which is very complicated from the experimental point of view.

In Refs. [3, 4, 5], a model of nucleons in terms of meson-baryon bound state fluctuations has been developed. This model describes well the $\bar{d}-\bar{u}$ and the $\bar{d} / \bar{u}$ asymmetries in the nucleon sea as measured by the E866 Collaboration [6]. The model also describes qualitatively the $s-\bar{s}$ asymmetry in nucleons observed in a recent global analysis of Deep Inelastic Scattering (DIS) data in nucleons [7]. Moreover, it is interesting to note that this model provides a consistent scheme to generate non-perturbative sea quark and gluon distributions at a low $Q^{2}$ scale. Let us remark that, as already noted by several authors [8], these non-perturbative, valence-like, sea quark and gluon distributions at the low $Q^{2}$ input scale are needed to fit DIS data.

The above facts indicate that this could be a sensible approach to the problem of having a low $Q^{2}$ model of hadrons. In this work, we will extend the model to pions and kaons and we shall explore the feasibility of extracting information on the low $Q^{2}$ structure of kaons within this scheme.

The paper is organized as follows. In the next section we will study the low $Q^{2}$ wave-function of pions and kaons. In section 3, we shall try to extract information on the kaon's pdf in terms of the model and the available experimental data and section 1 is devoted to conclusions and further discussion. 


\section{Low $Q^{2}$ wave-functions of pions and kaons}

To start with, let us consider the $\pi^{-}$and $K^{-}$. Their low $Q^{2}$ wave-functions can be expanded as

$$
\begin{aligned}
\left|\pi^{-}\right\rangle & =a_{0}^{\pi}\left|\pi_{0}^{-}\right\rangle+a_{1}^{\pi}\left|\pi^{-} g\right\rangle+a_{2}^{\pi}\left|K^{\circ} K^{-}\right\rangle \\
\left|K^{-}\right\rangle & =a_{0}^{K}\left|K_{0}^{-}\right\rangle+a_{1}^{K}\left|K^{-} g\right\rangle+a_{2}^{K}\left|\overline{K^{\circ}} \pi^{-}\right\rangle,
\end{aligned}
$$

in terms of hadron-like Fock state fluctuations. In the above expresions we have neglected higher order contributions involving heavier mesons. These fluctuations should be far off-shell and they can be safely ignored at this point. Coefficients in the expansions of eqs. (1) are constrained by probability conservation, $\sum_{i}\left(a_{i}^{M}\right)^{2}=1$.

Similar expresions can be obtained straighforwardly for $\pi^{+}, K^{+}$and neutral kaons. Neutral pions, however, have a different structure than the charged ones because they can also fluctuate to $\left|\pi^{+} \pi^{-}\right\rangle$Fock states (see discussion in Ref. [5]).

Hadrons in the Fock state fluctuations on the RHS of eqs. (11) are assumed to be formed only by constituent valence quarks or valons, in the terminology of Ref. [9]. Fluctuations themselves are responsible for the non-perturbative-intrinsic- sea, which should provide the necessary binding among constituent quarks to form hadrons (5, [10]. This means that, at a low $Q^{2}$ scale, hadrons are made of constituent valons plus intrinsic quark-antiquark pairs and gluons. At this point it is important to note that valence quark densities at the low $Q^{2}$ scale have contributions not only from valons in the bare meson state, but also from the fluctuations themselves. These fluctuations give rise also to the so called intrinsic sea of $q \bar{q}$ pairs and gluons.

DGLAP evolution to higher $Q^{2}$ generates the perturbative -extrinsic- sea of $q \bar{q}$ pairs and gluons. In this sense, evolution to higher $Q^{2}$ should mostly resolve the structure of valons themselves [9].

Let us call $Q_{v}^{2}$ the scale at which the hadron wave-function can be consistently written as a hadron-like Fock state expansion as those of eq. (1). Then, at the scale $Q_{v}^{2}$, parton distribution functions are given by

$$
\begin{aligned}
& \bar{u}_{\pi}(x)=d_{\pi}(x)=\left(a_{0}^{\pi}\right)^{2} v_{\pi}(x)+\left(a_{1}^{\pi}\right)^{2} P_{\pi g} \otimes v_{\pi}+\left(a_{2}^{\pi}\right)^{2} P_{K K} \otimes v_{K} \\
& s_{\pi}(x)=\bar{s}_{\pi}(x)=\left(a_{2}^{\pi}\right)^{2} P_{K K} \otimes v_{s / K} \\
& g_{\pi}(x)=\left(a_{1}^{\pi}\right)^{2} P_{g \pi}(x)
\end{aligned}
$$

for pions, and

$$
\begin{aligned}
\bar{u}_{K}(x) & =\left(a_{0}^{K}\right)^{2} v_{\bar{u} / K}(x)+\left(a_{1}^{K}\right)^{2} P_{K g} \otimes v_{K}+\left(a_{2}^{K}\right)^{2} P_{\pi K} \otimes v_{\pi} \\
s_{K}(x) & =\left(a_{0}^{K}\right)^{2} v_{s / K}(x)+\left(a_{1}^{K}\right)^{2} P_{K g} \otimes v_{s / K}+\left(a_{2}^{K}\right)^{2} P_{K \pi} \otimes v_{s / K} \\
d_{K}(x) & =\left(a_{2}^{K}\right)^{2} P_{\pi K} \otimes v_{\pi} \\
\bar{d}_{K}(x) & =\left(a_{2}^{K}\right)^{2} P_{K \pi} \otimes v_{K} \\
g_{K}(x) & =\left(a_{1}^{K}\right)^{2} P_{g K}(x)
\end{aligned}
$$


for kaons. In eqs. (2) and (31)

$$
P_{M M^{\prime}} \otimes v_{q / M} \equiv \int_{x}^{1} \frac{d y}{y} P_{M M^{\prime}}(y) v_{q / M}\left(\frac{x}{y}\right)
$$

is the probability density of the non-perturbative contribution to the parton distribution, coming from the $\left|M M^{\prime}\right\rangle$ fluctuation [4, 5]. $v_{q / M}$ is the $q$-flavored valon distribution in the meson $M$. A brief description about how to obtain the $\left|P_{M M^{\prime}}\right\rangle$ probability densities is given in the Appendix. In order to arrive to eqs. (2) and (3), we have assumed

$$
\begin{aligned}
v_{\pi}(x) & \equiv v_{\bar{u} / \pi^{-}}(x)=v_{d / \pi^{-}}(x)=v_{u / \pi^{+}}(x)=v_{\bar{d} / \pi^{+}}(x) \\
v_{K}(x) & \equiv v_{\bar{u} / K^{-}}(x)=v_{d / K^{0}}(x)=v_{\bar{d} / \bar{K}^{\circ}}(x)=v_{u / K^{+}}(x) \\
v_{s / K} & \equiv v_{s / K^{-}}(x)=v_{\bar{s} / K^{+}}(x)=v_{s / K^{\circ}}(x)=v_{\bar{s} / \bar{K}^{\circ}}(x)
\end{aligned}
$$

which follow from isospin invariance.

Similar expresions to those of eqs. (2) and (3) can be easily obtained for the $\pi^{+}$ and $K^{+}$parton distribution functions.

It must be noted that there are no fluctuations containing neutral pions in the expansions of eq. (四). In fact, as discussed in Ref. [5], pions cannot fluctuate to a $\left|\pi^{ \pm, o} \pi^{\circ}\right\rangle$ Fock state. This would imply the formation of a neutral in-pion state which can rapidly decay to a gluon. Actually, an unflavored structure like $v_{q} \bar{q}$ has the quantum numbers of a gluon, giving rise to the $\left|\pi^{ \pm, \circ} g\right\rangle$ Fock state. Consequently, the first contribution to the intrinsic $q \bar{q}$ sea in pions arises in the strange sector. Intrinsic $u \bar{u}$ and $d \bar{d}$ sea quarks, however, must be considered in neutral pions due to contributions coming from the $\left|\pi^{+} \pi^{-}\right\rangle$fluctuation [5].

Similarly, kaons cannot fluctuate to $\left|K^{ \pm, \circ} \pi^{\circ}\right\rangle$ bound states, which in turn are the origin of the $\left|K^{ \pm, \circ} \mathrm{g}\right\rangle$ fluctuation. Consequently, in kaons, the first contribution to the intrinsic $q \bar{q}$ sea arises in the light sector. In fact, the $\left|\bar{K}^{\circ} \pi^{-}\right\rangle\left(\left|K^{\circ} \pi^{+}\right\rangle\right)$fluctuation gives rise to the $d \bar{d}$ intrinsic sea of the $K^{-}\left(K^{+}\right)$while the $\left|K^{+} \pi^{-}\right\rangle\left(\left|K^{-} \pi^{+}\right\rangle\right)$one originates the $u \bar{u}$ intrinsic sea in the $\bar{K}^{\circ}\left(K^{\circ}\right)$ respectively. It is interesting to note also that there is no $s \bar{s}$ intrinsic sea in kaons. The reason is that fluctuations to $\left|K^{ \pm, \circ} \phi\right\rangle$, which could potentially originate the strange intrinsic sea, also contribute to the intrinsic gluon distribution due to the rapid anihilation of the unflavored $v_{s / K} \bar{s}$ structure. However, this contribution must be smaller than those coming from the anihilation of a light valon with a light antiquark.

A careful look at the eqs. (2) and (3) reveals other interesting properties of the low $Q^{2}$ wave-functions of pions and kaons. First of all, if the $v_{\pi}, v_{K}$ and $v_{s / K}$ valon distributions are known, the entire structure of pions and kaons is determined up to the coefficients giving the probability of each individual fluctuation. A second interesting property is that the intrinsic strange sea in pions is entirely determined by the strange valon distribution in kaons. Then, by using the convolution theorem 
applied to Mellin transforms we can formally invert the second expression in eq. (2) to obtain $v_{s / K}$ :

$$
v_{s / K}(n)=\frac{s_{\pi}(n)}{\left(a_{2}^{\pi}\right)^{2} P_{K K}(n)}
$$

where $f(n)$ is the Mellin transform of $f(x)$. Notice that the above equation not only determines the shape of $v_{s / K}$, but also fixes the coefficient $a_{2}^{K}$, since the strange valon distribution must be normalized to one.

Once $v_{s / K}(x)$ is known, the $v_{K}(x)$ distribution become fixed by momentum conservation, thus

$$
v_{K}(x)=v_{s / K}(1-x) .
$$

Then, by only measuring the strange quark distribution in pions, the valon probability densities in kaons become determined. Now, by measuring the $\bar{u}_{\pi}$ and $g_{\pi}$ we can determine the remaining $v_{\pi}$ through

$$
v_{\pi}(n)=\frac{\bar{u}_{\pi}(n)-\left(a_{2}^{\pi}\right)^{2} P_{K K}(n) v_{K}(x)}{\left(a_{0}^{\pi}\right)^{2}+\left(a_{1}^{\pi}\right)^{2} P_{\pi g}(n)}
$$

where we have used again the convolution theorem applied to Mellin transforms. Since the coefficients $a_{1}^{\pi}$ and $a_{2}^{\pi}$ are fixed once the strange and gluon distributions in pions are measured, then there are no free parameters in eq. (8) due to probability conservation.

To extract the shape of the required valon distributions and the value of the coefficients $a_{i}^{\pi}(i=1,2,3)$, data on $\pi^{ \pm}-$nucleon D-Y dilepton cross sections must be fitted using the formulae presented in eqs. (2). On this respect, it is worth to note that $\pi p \rightarrow \gamma X$ cross sections are dominated by $q g$ scattering, then they are sensitive to the gluon distribution. On the other hand, the difference of cross sections $\sigma\left(\pi^{-} p\right)-$ $\sigma\left(\pi^{+} p\right)$ is dominated by $q \bar{q}$ anihilation, allowing in this way the determination of quark distributions in pions. Furthermore, in Ref. [12] it has been argued that adequate linear combinations of $\pi^{ \pm}-$nucleon $\mathrm{D}$-Y cross sections should allow the determination of valence densities in pions, independently of sea quark distributions. Having the gluon and valence quark distributions, the sea quark densities can be known. Thus, the determination of the valon densities $v_{\pi}, v_{K}$ and $v_{s / K}$ and the parameters $a_{i}^{\pi}$ can be achieved.

In practice, however, some sort of iterative procedure is needed to determine $v_{\pi}$, $v_{K}$ and $v_{s / K}$ from experimental data since $v_{\pi}$ enters in the calculation of the $P_{\pi g}$ and $P_{K K}$ probability densities.

At this point some remarks are in order. As we have shown above, the valon distributions and parameters entering in the pion wave-function can be determined from measurements of the pion pdf. Having this information, we can proceed to determine the kaon pdf. To this end, however, some experimental input on kaon parton distributions is needed. The $\bar{u}_{K}$ distribution can be measured in $K^{-}$-nucleon 
D-Y experiments, then we can use the relationship in the first line of eqs. (3) to fix the coefficients $a_{i}^{K}(i=1,2,3)$ in the kaon wave-function. Since $v_{K}$ and $v_{s / K}$ have been previously determined, then the kaon pdf become completely fixed. This will be done in the next section.

\section{$3 \quad$ Kaon parton distributions}

The existing parametrizations of pion pdf have been obtained assuming an $S U(3)_{\text {flavor }}$ symmetric sea. Furthermore, in most cases the $q \bar{q}$ sea has been generated perturbatively through DGLAP evolution [13, 14. Then it is not possible to determine the valon distributions $v_{\pi}, v_{K}$ and $v_{s / K}$ from present parametrizations. This will require to reanalyse the existing data on $\pi^{ \pm}-$nucleon dilepton and prompt photon production in terms of the low $Q_{v}^{2}$ parametrizations of eqs. (2). Consequently, for the moment we refrain from any attempt of determining the valon distributions from experimental data. Instead, we will use the valon distribution in pions and kaons proposed in Ref. 9],

$$
\begin{aligned}
v_{\pi}(x) & =1 \\
v_{K}(x) & =\alpha_{K} x^{a-1}(1-x)^{b-1} \\
v_{s / K}(x) & =\alpha_{K} x^{b-1}(1-x)^{a-1},
\end{aligned}
$$

where $a$ and $b$ are related by $a / b=m_{l} / m_{h} \sim 2 / 3, m_{l}$ and $m_{h}$ are the light and strange valon masses in kaons and $\alpha_{K}$ is a normalization constant.

These simple valon parametrizations would allow us to have both an idea of the feasibility of this method to extract kaon's pdf, as well as to have some knowledge of the kaon low $Q_{v}^{2}$ structure.

To obtain the values of the $a_{i}^{K}$ coefficients in the kaon wave-function, data on $\bar{u}_{k} / \bar{u}_{\pi}$ by the NA3 Collaboration [2] were fitted using

$$
\frac{\bar{u}_{K}(x)}{\bar{u}_{\pi}(x)}=\frac{\left(a_{0}^{K}\right)^{2} v_{K}(x)+\left(a_{1}^{K}\right)^{2} P_{K g} \otimes v_{K}(x)+\left(a_{2}^{K}\right)^{2} P_{\pi K} \otimes v_{\pi}(x)}{\bar{u}_{\pi}(x)},
$$

together with the valon distributions of eqs. (9). Notice that the fitting function has only two free parameters due to probability conservation.

In order to do the fits we assumed that the $\bar{u}_{K} / \bar{u}_{\pi}$ ratio is independent of $Q^{2}$. We recognize that this assumption could be questionable, but given the large error bars of the NA3 data, and the uncertainty in the valons distributions, fits are hardly sensitive to QCD evolution.

In these fits we also used two different $\bar{u}_{\pi}$ distributions; the GRV-P [14] parametrization at the low $Q_{v}^{2}$ and the $\bar{u}_{\pi}$ distribution obtained by means of a Monte Carlo model of hadrons in Ref. [15. This enables us to test the sensibility of the model to the shape of the $\bar{u}_{\pi}$ distribution. 
Table 1: Parameters in the $\bar{u}_{K} / \bar{u}_{\pi}$ fitting function.

\begin{tabular}{c|c|c|c}
\hline \hline$x \bar{u}_{\pi}(x)$ & $\left(a_{0}^{K}\right)^{2}$ & $\left(a_{1}^{K}\right)^{2}$ & $\left(a_{2}^{K}\right)^{2}$ \\
\hline GRV-P [14 & $0.392 \pm 0.050$ & $0.101 \pm 0.059$ & $0.507 \pm 0.077$ \\
$2.3 x^{1.1}(1-x)$ [15] & $0.646 \pm 0.083$ & $0.177 \pm 0.097$ & $0.177 \pm 0.127$ \\
\hline \hline
\end{tabular}

The results of the fits are displayed in Fig. (1) and in Table (11). All the fits were done using $a=1.5$ and $b=2.25$ in the $v_{K}$ distribution of eqs. (9). As can be seen in the Figure, the main effect of using a different $\bar{u}_{\pi}$ distribution is in the low $x(<0.2)$ region. But in this region, the pion valence distribution is not well known.

A noticeable effect is also evident in the value of the $a_{i}^{K}$ coefficients (see Table (11)). In fact, using the GRV-P parametrization, the probability of the $\left|\bar{K}^{\circ} \pi^{-}\right\rangle$fluctuation of the kaon is bigger than the probability of the $\left|K_{0}\right\rangle$ state, opposite to intuition. On the other hand, using the $\bar{u}_{\pi}$ distribution of Ref. [15], one gets $a_{1}^{K} \sim a_{2}^{K}$ and $a_{0}^{K}>a_{1}^{K}$, as expected. Given the uncertainties coming mainly from the shape of the valon distributions, this facts have no special meaning regarding valence distributions in pions. However, the general scheme proposed seems to be significant in order to extract kaon's pdf.

In Fig. (2) we display the $\bar{u}_{K}$ distribution obtained from fits using the $\bar{u}_{\pi}$ parametrization of Ref. [15] in comparison to the $\bar{u}_{\pi}$ distribution itself. As expected, the $\bar{u}_{K}$ probability density is peaked at lower $x$ than the one of the pion, indicating that light valence quarks in kaons carry in average less momentum than valence quark in pions.

In Fig. (3) we show the full set of valence and intrinsic $q \bar{q}$ and gluon distributions in kaons at the low $Q_{v}^{2}$ scale. These distributions were calculated using the coefficients in the second row of Table (四) together with eqs. (3), (4) and (9).

\section{Conclusions}

The study of the low $Q^{2}$ wave-function of hadrons is an important issue for several reasons. The first one is related to the origin of the valence-like distributions needed to fit the experimental data on hadron's pdf. This fact, which has already been noted repeatedly by several authors in the case of nucleon's pdf, is also important in order to determine the pion pdf (see, for instance, the last Ref. [13]). Furthermore, for pions there is no theoretical input regarding what initial valence-like sea quark or gluon distributions are needed in order to describe experimental data. Notice that most of the pion's pdf available in the literature are determined using a $S U(3)_{\text {flavor }}$ symmetric sea, which is surely incorrect.

The second important reason concerns the kaon structure itself. As a matter of fact, the experimental information one can get on the kaon structure is only on the 
light valence quark distribution. The measurement of the strange and even sea quark distributions in kaons is not possible due to practical reasons. Actually, strange and sea quarks only contribute to the total D-Y dilepton cross section through valence-sea and sea-sea $q \bar{q}$ anihilation. Then their contributions are small and cannot be easily separated. Thus, we must rely on other methods to obtain information on the kaon structure. It is worth to stress that the knowledge of the kaon structure is not only important by itself, but also because kaons are commonly used in many experiments and their pdf are needed to calculate the cross sections of the produced particles.

In this work we addressed the problem of the low $Q^{2}$ structure of pions and kaons. We have shown, by using a hadron-like Fock state expansion of the pion and kaon wave-functions, that the pion and kaon structure are related one another. This enables one to determine the complete structure of kaons from a minimal set of measurements of the pion and kaon pdf. In fact, by exploiting the relationship among the pion and kaon low $Q^{2}$ wave-functions, the kaon structure can be completely determined by measuring pion structure and the $\bar{u}_{K} / \bar{u}_{\pi}$ ratio. We would like to remark, however, that a confident determination of kaon pdf requires a reanalysis of the pion data in terms of the model presented here.

\section{Acknowledgments}

J.M. is partially supported by COLCIENCIAS, the Colombian Agency for Science and Technology, under Contract No. 242-99.

\section{Appendix: In-meson hadron distributions}

The meson probability density $P_{M M^{\prime}}(x)$ in the $\left|M M^{\prime}\right\rangle$ fluctuation has been calculated in Refs. [4, 5n. It is given by

$$
P_{M M^{\prime}}(x)=\int_{0}^{1} \frac{d y}{y} \int_{0}^{1} \frac{d z}{z} F(y, z) R(x, y, z)
$$

with

$$
\begin{aligned}
F(y, z) & =\beta y v_{q}(y) z q^{\prime}(z)(1-y-z)^{a} \\
R(x, y, z) & =\alpha \frac{y z}{x^{2}} \delta\left(1-\frac{y+z}{x}\right) .
\end{aligned}
$$

In eqs. (12), $v_{q}$ and $q^{\prime}$ are the valon and the quark or antiquark distributions which will form the meson $M$ in the $\left|M M^{\prime}\right\rangle$ fluctuation. The $q^{\prime}$ distribution is generated through the gluon emission from a valon followed by the $q^{\prime}-\bar{q}^{\prime}$ pair creation, which are basic processes in QCD. Thus its distribution is given by [3]

$$
q^{\prime}(x)=\bar{q}^{\prime}(x)=N \frac{\alpha_{s t}^{2}\left(Q_{v}^{2}\right)}{(2 \pi)^{2}} \int_{x}^{1} \frac{d y}{y} P_{q g}\left(\frac{x}{y}\right) \int_{y}^{1} \frac{d z}{z} P_{g q}\left(\frac{y}{z}\right) v_{q}(z),
$$


where $P_{q g}(z)$ and $P_{g q}(z)$ are the Altarelli-Parisi splitting functions [11] given by

$$
P_{g q}(z)=\frac{4}{3} \frac{1+(1-z)^{2}}{z}, \quad P_{q g}(z)=\frac{1}{2}\left(z^{2}+(1-z)^{2}\right) .
$$

It is worth to note that the only scale dependence appearing in eq. (13) arises through $\alpha_{s t}\left(Q^{2}\right)$. Since the valon scale is tipically of the order of $Q_{v}^{2} \sim 0.64 \mathrm{GeV}^{2}$ [9], then the $q^{\prime}-\bar{q}^{\prime}$ pair creation can be safely evaluated perturbatively because $\alpha_{s t}^{2} /(2 \pi)^{2}$ is still sufficiently small. The normalization constants $\alpha, \beta$ and $N$ in eqs. (12) and (13) contribute to the global normalization coefficient of the corresponding Fock state fluctuation in the expansions of eqs. (1).

Momentum conservation also requires

$$
P_{M M^{\prime}}(x)=P_{M^{\prime} M}(1-x),
$$

a condition which relates the in-meson $M$ and $M^{\prime}$ probability densities. Additionally, hadrons in the $\left|M M^{\prime}\right\rangle$ fluctuation must be correlated in velocity in order to form a bound state. This imply that

$$
\frac{\left\langle x P_{M M^{\prime}}(x)\right\rangle}{m_{M}}=\frac{\left\langle x P_{M^{\prime} M}(x)\right\rangle}{m_{M}^{\prime}},
$$

fixing in this way the exponent $a$ in eqs. (12). Notice also that $P_{g M}$ is calculated from the "recombination" of an antiquark with a valon of the same flavor [5. Then, formally, the $P_{g M}$ corresponds to the $\pi^{\circ}$ distribution in a hypothetical $\left|M \pi^{\circ}\right\rangle$ fluctuation.

\section{References}

[1] R.G. Badalyan and G.R. Gulkanyan, Sov. J. Nucl. Phys. 50, 115 (1989); N.N. Badalyan, R.G badalyan and H.R Gulkanian, Z. Phys. C42, 69 (1989).

[2] J. Badier et al., Phys. Lett. B93, 354 (1980).

[3] H.R. Christiansen and J. Magnin, Phys. Lett. B445, 8 (1998).

[4] J. Magnin and H.R. Christiansen, Phys. Rev. D61, 054006 (2000).

[5] H.R. Christiansen and J. Magnin, hep-ph/0003088.

[6] E.A. Hawker et al., Phys. Rev. Lett. 80, 3715 (1998); J.C. Peng et al., Phys. Rev. D58, 092004 (1998).

[7] V. Barone, C. Pascaud and F. Zomer, Eur. Phys. J. C12, 243 (2000). 
[8] M. Glück, E. Reya and A. Vogt, Z. Phys. C53, 127 (1992), C67, 433 (1995); J. Botts et al., Phys. Lett. B304, 159 (1993); H.L. Lai et al., Phys. Rev. D51, 4763 (1995); A.D. Martin, W.J. Stirling and R.G. Roberts, Phys. Lett. B354, 155 (1995).

[9] R.C. Hwa, Phys. Rev D22, 1553 (1980).

[10] S.J. Brodsky, C. Peterson and N. Sakai, Phys. Rev. D23, 2745 (1981).

[11] G. Altarelli and G. Parisi, Nucl. Phys. B126, 298 (1977).

[12] J.T. Londergan, G.Q. Liu, E.N. Rodionov and A.W. Thomas, Phys. Lett. B361, 110 (1995).

[13] P. Aurenche et al., Phys. Lett. B233 517 (1989); P.J. Sutton, A.D. Martin, R.G. Roberts and W.J. Stirling, Phys. Rev. D45, 2349 (1992); M. Glück, E. Reya and I. Schienbein, Eur. Phys. J. C10, 313 (1999).

[14] M. Glück, E. Reya and A. Vogt, Z. Phys. C53, 651 (1992).

[15] A. Edin and G. Ingelman, Phys. Lett. B423, 402 (1998). 


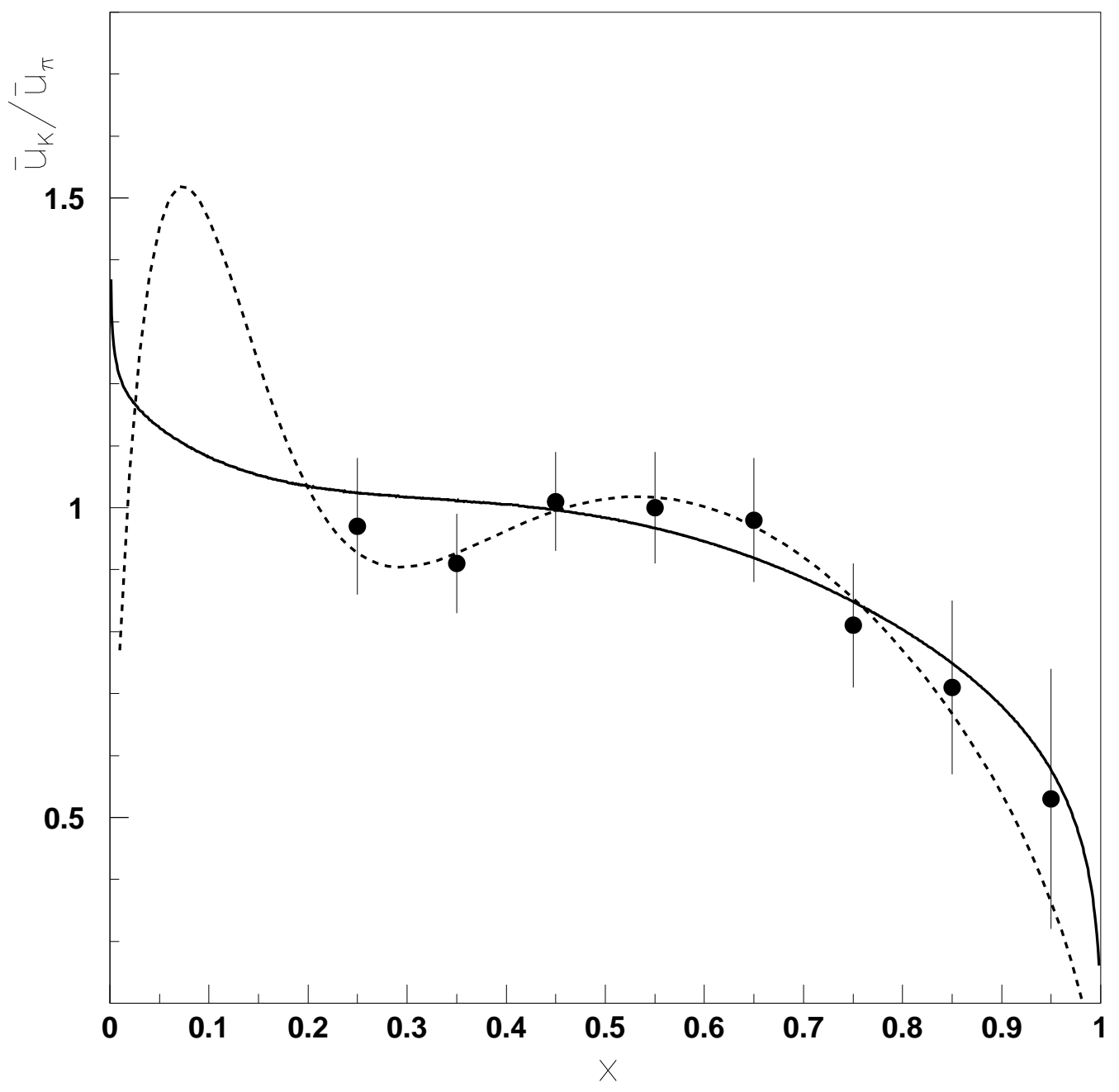

Figure 1: $\bar{u}_{K} / \bar{u}_{\pi}$ as a function of $\mathrm{x}$. Data is from Ref. [2]. Dashed line is the fit using the $\bar{u}_{\pi}$ distribution of Ref. [14], the solid line is the fit using the $\bar{u}_{\pi}$ distribution given in Ref. [15]. 


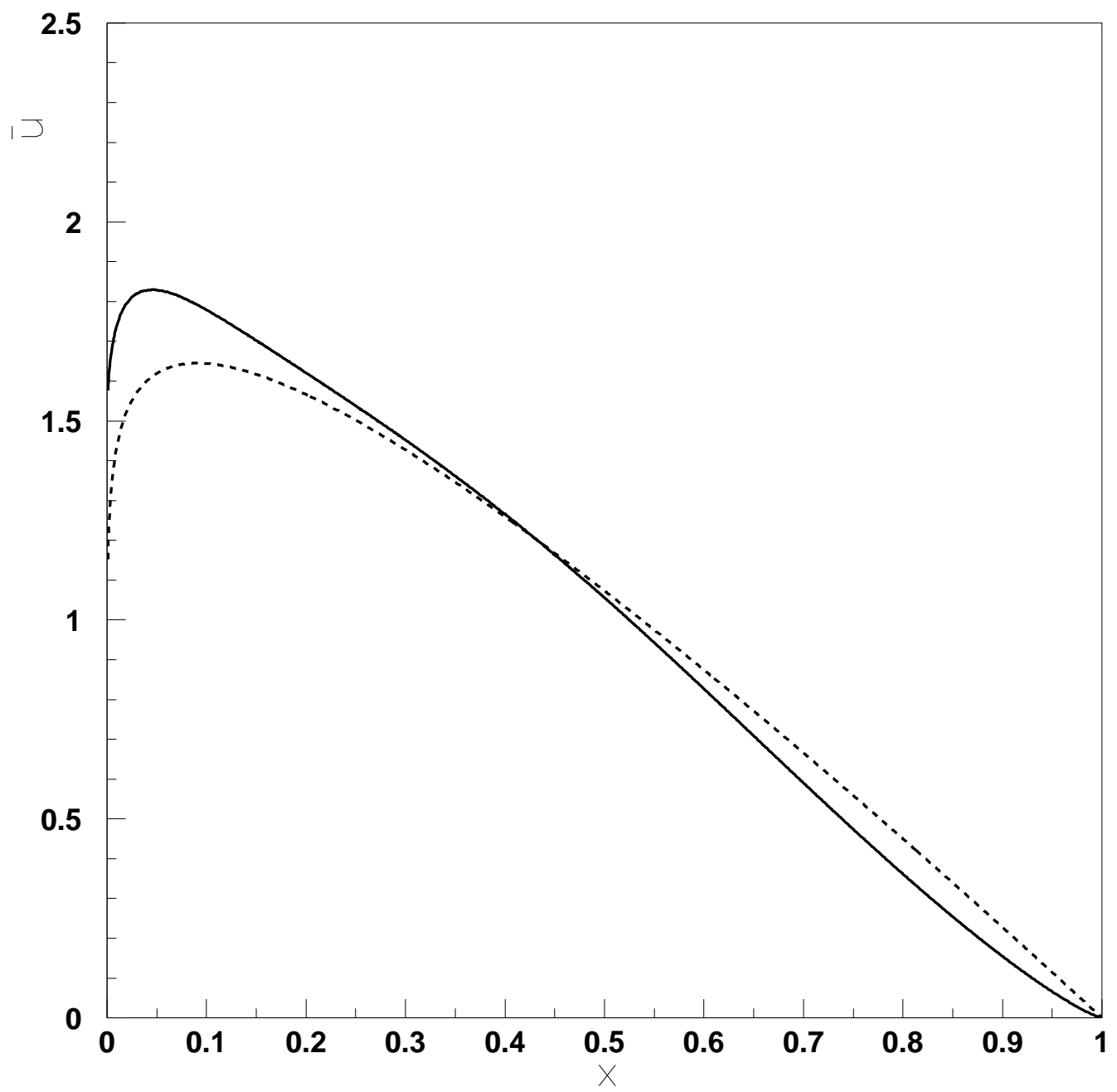

Figure 2: $\bar{u}_{K}$ (solid line) compared to the $\bar{u}_{\pi}$ (dashed line) distribution as function of $\mathrm{x}$ at the low $Q_{v}^{2}$ scale. The $\bar{u}_{K}$ distribution comes from the coefficients in the second

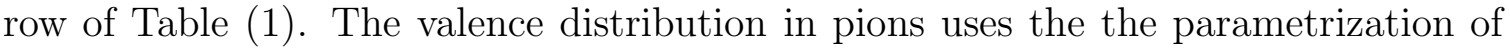
Ref. [15]. 

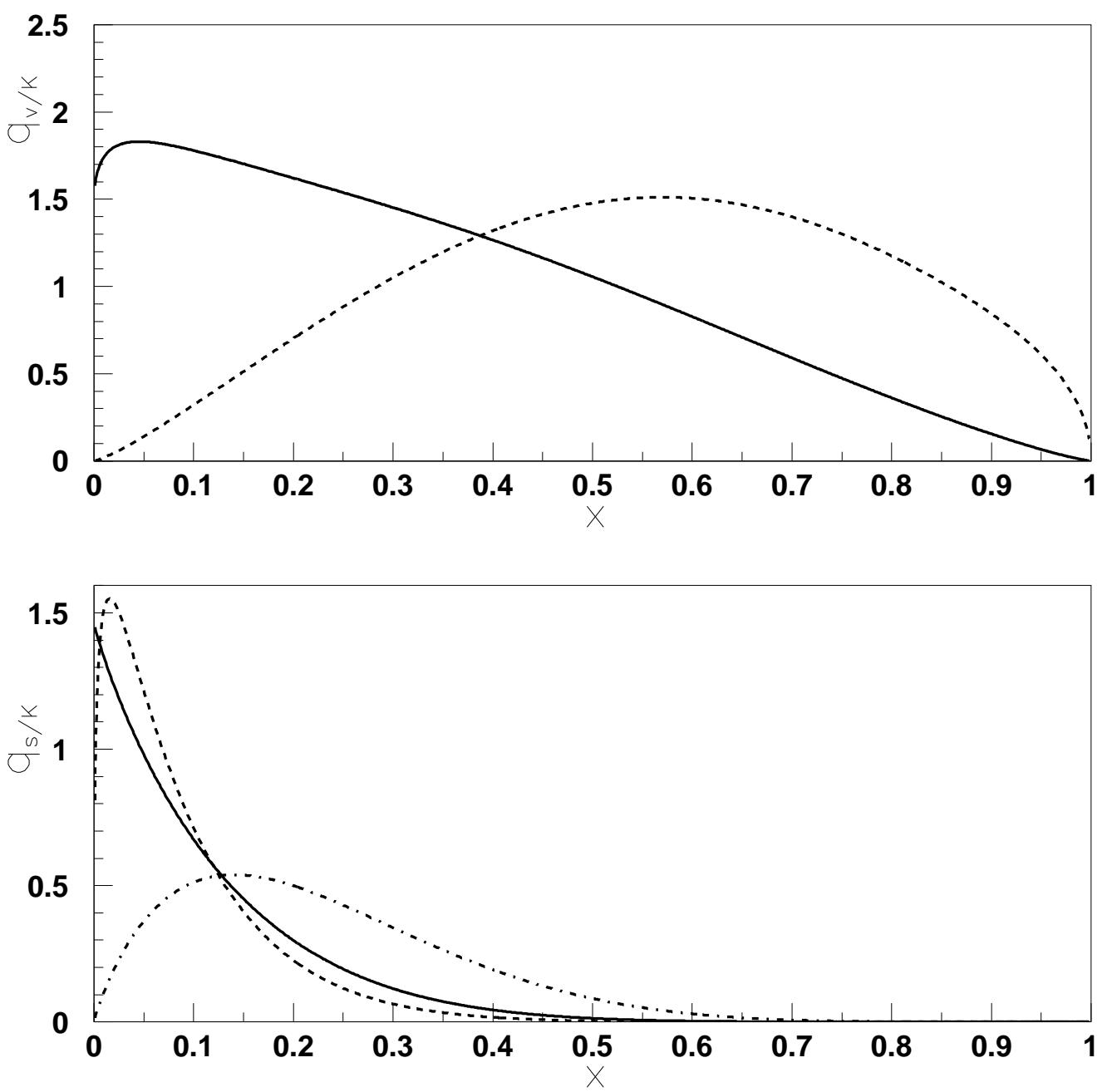

Figure 3: $K^{-}$parton distributions at the $Q_{v}^{2}$ scale as a function of $x$ obtained from fits using the $\bar{u}_{\pi}$ parametrization given in Ref. [15]. Upper: $\bar{u}_{K}$ (solid line) and $s_{K}$ (dashed line) distributions. Lower: $d_{K}$ (solid line), $\bar{d}_{K}$ (dashed line) and $g_{K}$ (point dashed) distributions. 